\title{
Modern Software for Computer Modeling in Quantum Chemistry and Molecular Dynamics
}

\author{
Marina V. Malyshkina ${ }^{1,2}$ and Alexander S. Novikov ${ }^{2, *(D)}$ \\ 1 Presidential Physics and Mathematics Lyceum No. 239, Kirochnaya St., 8, Bldg. A, 191028 Saint Petersburg, \\ Russia; mar_mal05@mail.ru \\ 2 Infochemistry Scientific Center, ITMO University, Kronverksky Pr., 49, Bldg. A, 197101 Saint Petersburg, Russia \\ * Correspondence: novikov@itmo.ru
}

check for updates

Citation: Malyshkina, M.V.; Novikov, A.S. Modern Software for Computer Modeling in Quantum Chemistry and Molecular Dynamics. Compounds 2021, 1, 134-144. https://doi.org/ 10.3390/compounds1030012

Academic Editor: Juan Mejuto

Received: 31 August 2021

Accepted: 2 November 2021

Published: 16 November 2021

Publisher's Note: MDPI stays neutral with regard to jurisdictional claims in published maps and institutional affiliations.

Copyright: (c) 2021 by the authors. Licensee MDPI, Basel, Switzerland. This article is an open access article distributed under the terms and conditions of the Creative Commons Attribution (CC BY) license (https:// creativecommons.org/licenses/by/ $4.0 /)$.

\begin{abstract}
The most popular modern programs for quantum chemical and molecular dynamics (classical, ab initio, and QM/MM) calculations, which are relevant for the investigation of nature and various properties of different molecules and periodic chemical systems such as nanotubes, surfaces and films, polymers, and crystalline solids, are highlighted and briefly discussed.
\end{abstract}

Keywords: quantum chemistry; computational chemistry; molecular dynamics; modeling; open-source software; proprietary software

In the modern world, computer modeling can quite successfully compete with experimental methods (and compliment them) in many areas of science and technology. The quantum and computational chemistry as well as molecular dynamics are no exception, and currently there are many open-source and proprietary program solutions for the investigation of nature and various properties of different molecules and periodic chemical systems such as nanotubes, surfaces and films, polymers, and crystalline solids. In this mini-review, we would like to highlight and briefly discuss some of such programs for computer modeling in chemistry and materials science. A summary of the programming language, type of license, and possibility to calculate NMR, as well as vibrational spectra and thermochemistry, for all discussed software for computer modeling in quantum chemistry and molecular dynamics is given in Table 1.

ABINIT [1] is an open-source suite that was developed for programs of physics, chemistry, materials science, and others. ABINIT realizes implementation of density functional theory (DFT) by performing the solution of the Kohn-Sham equations. The equations describe the electrons in a chemical system, the presentation of which is implemented in a plane wave basis set. The energy minimum is determined through usage of a self-consistent conjugate gradient method. Computational efficiency and high speed are achieved by fast Fourier transforms, and effective core potentials (aka pseudopotentials) to describe core (non-valence) electrons. ABINIT implements an alternative to standard norm-conserving pseudopotentials. The application of the projector augmented-wave method is possible. In addition to total electronic energy, stresses and forces are calculated; moreover, geometry optimizations and ab initio molecular dynamics are capable of being performed and calculated.

Aces II and III [2] are computational chemistry packages that were elaborated for implementing high-level ab initio quantum chemical calculations. Approaching manybody techniques, for instance, cluster techniques, in particular, to treat electron correlation in details and many-body perturbation theory, allows the precise prediction of atomic and molecular energies in addition to properties.

BerkeleyGW [3] is a code, specialized on excited states, for many-body perturbation theory. The BerkeleyGW package implements the solution for optical properties of materials and quasiparticle excitations by performing the GW plus Bethe-Salpeter equation 
(GW-BSE) method and the GW method. The latest BerkeleyGW can be applied to study systems up to a few thousand atoms.

Table 1. Summary of the programming language, type of license, and possibility to calculate NMR, as well as vibrational spectra and thermochemistry, for all discussed software for computer modeling in quantum chemistry and molecular dynamics.

\begin{tabular}{|c|c|c|c|c|c|}
\hline Package & Language & License & NMR & Vib. Spec. & Thermo. \\
\hline 1 ABINIT & Fortran & Free, GPL & + & + & + \\
\hline 2 ACES & Fortran, C++ & Free, GPL & + & + & + \\
\hline 3 BerkeleyGW & Fortran & Free, GPL & + & + & + \\
\hline 4 BigDFT & Fortran & Free, GPL & - & + & + \\
\hline 5 BrianQC & $\mathrm{C}++, \mathrm{CUDA}$ & Commercial & + & + & + \\
\hline 6 CASTEP & Fortran 95, Fortran 2003 & Academic, commercial & + & + & + \\
\hline 7 COLUMBUS & Fortran & Academic & - & + & + \\
\hline $8 \mathrm{CP} 2 \mathrm{~K}$ & Fortran 95 & Free, GPL & + & + & + \\
\hline 9 CPMD & Fortran & Academic & + & + & + \\
\hline 10 CRYSTAL & Fortran & $\begin{array}{l}\text { Academic (UK), } \\
\text { Commercial (IT) }\end{array}$ & - & + & + \\
\hline 11 DACAPO & Fortran & Free, GPL & - & + & - \\
\hline 12 Dalton & Fortran & Free, LGPL & + & + & + \\
\hline 13 deMon2k & Fortran & Academic, commercial & + & + & + \\
\hline 14 DMol3 & Fortran 90 & Commercial & + & + & + \\
\hline $15 \mathrm{DP}$ & Fortran 90, C & Free, LGPL & - & - & - \\
\hline 16 ErgoSCF & $\mathrm{C}++$ & Free, GPL & - & - & - \\
\hline 17 Exabyte.io & Python & Cloud, Free Tier & - & + & + \\
\hline 18 FHI-aims & Fortran & Academic, commercial & + & + & + \\
\hline 19 FPLO & Fortran 95, C++, Perl & Commercial & - & - & - \\
\hline 20 GAMESS (UK) & Fortran & $\begin{array}{l}\text { Academic UK, } \\
\text { Commercial }\end{array}$ & + & + & + \\
\hline 21 GAMESS (US) & Fortran & Academic & + & + & + \\
\hline 22 Gaussian & Fortran & Commercial & + & + & + \\
\hline 23 GPAW & Python, C & Free, GPL & + & + & + \\
\hline 24 HORTON & Python, C++ & Free, GPL & - & + & + \\
\hline 25 HyperChem & $\mathrm{C}++$ & Commercial & + & + & + \\
\hline 26 JDFTx & $\mathrm{C}++$ & Free, GPL & + & + & + \\
\hline 27 Maple & Maple, C, Fortran, Python & Commercial & - & - & - \\
\hline 28 MOLCAS & Fortran, C, C++, Python, Perl & Academic, commercial & + & + & + \\
\hline 29 MOLGW & Fortran & Free, GPL & - & - & - \\
\hline 30 MOLPRO & Fortran & Commercial & + & + & + \\
\hline 31 MOPAC & Fortran & Academic, commercial & - & + & + \\
\hline $32 \mathrm{MPQC}$ & $\mathrm{C}++$ & Free, LGPL & + & + & + \\
\hline $33 \mathrm{MRCC}$ & Fortran & Academic & + & + & + \\
\hline 34 NTChem & Unknown & Commercial & + & + & + \\
\hline 35 NWChem & Fortran $77, \mathrm{C}$ & Free, ECL v2 & + & + & + \\
\hline 36 ONETEP & Fortran 2003 & $\begin{array}{l}\text { Academic (UK), } \\
\text { Commercial }\end{array}$ & - & + & + \\
\hline 37 OpenAtom & Charm $++(\mathrm{C}++)$ & Academic & - & + & + \\
\hline 38 OpenMx & $\mathrm{C}$ & Free, GPL & + & + & + \\
\hline 39 ORCA & $\mathrm{C}++$ & Academic, commercial & + & + & + \\
\hline 40 PARSEC & Fortran & Free, GPL & - & - & - \\
\hline 41 PSI & C, C++, Python & Free, GPL & - & + & + \\
\hline 42 PyQuante & Python & Free, BSD & - & + & + \\
\hline 43 PySCF & Python & Free, BSD & + & + & + \\
\hline 44 QMCPACK (QMC) & $\mathrm{C}++$ & $\begin{array}{l}\text { Free, U. Illinois Open } \\
\text { Source }\end{array}$ & - & - & - \\
\hline 45 Quantum ESPRESSO & Fortan & Free, GPL & + & + & + \\
\hline 46 RMG & $\mathrm{C}, \mathrm{C}++$ & Free, GPL & - & + & + \\
\hline 47 SAMSON & $\mathrm{C}++$, Python & Free & - & - & - \\
\hline 48 SIESTA & Fortran 2003 & Free, GPL & - & - & - \\
\hline 49 VOTCA-ХTP & $\mathrm{C}++$ & Free, Apache License & - & - & - \\
\hline 50 Yambo & Fortran & Free, GPL & - & - & - \\
\hline
\end{tabular}


BigDFT [4] is an open-source software package, developed for physicists and chemists for innovative research of materials and macro-molecular systems at the nanoscale. In this software package implementation, DFT is realized by solution of the Kohn-Sham equations, a self-consistent direct minimization, and Davidson diagonalization methods. The Kohn-Sham equations describe the electrons in a material, expanded in a Daubechies wavelet basis set, and Davidson diagonalization methods determine the energy minimum on the potential energy surface. Computational efficiency is achieved with fast short convolutions and pseudopotentials to describe core (non-valent) electrons. BigDFT was among the first massively parallel density functional theory codes that benefited from graphics processing units (GPUs) with the usage of CUDA and then OpenCL languages.

BrianQC is a GPU module for Q-Chem [5]. As a Q-Chem software, it is able to calculate high angular momentum orbitals, and is highly efficient for simulating large molecules and quantum systems. BrianQC makes Q-Chem run on GPUs and speeds up DFT and Hartree-Fock (HF) single point, geometry optimization, and frequency calculations, as well as many other methods.

CASTEP [6] is a fully functional materials modelling code. It is based on a firstprinciples quantum mechanical description of electrons and nuclei (including usage of path-integral molecular dynamics). The CASTEP package is capable of simulation of an extensive range of materials properties such as vibrational properties, energetics, and electronic response properties and structure at the atomic level using density functional theory. Moreover, it has a great list of spectroscopic features, such as IR and Raman spectroscopies, core level spectra, and NMR, which are related directly to the experiment.

COLUMBUS [7] is a set of programs for high-level ab initio molecular electronic structure calculations. Specially, calculations on electronic ground and excited states of atoms and molecules were implemented by performing distinctive methods such as multi-reference average quadratic coupled-cluster (MR-AQCC), multireference averaged coupled-pair-functional (MR-ACPF), multiconfiguration self-consistent field (MCSCF), multireference configuration interaction with all single and double excitations (MR-CISD), and so on. COLUMBUS's unity of programs is available to select reference configurations in addition to typical classes of reference wave functions (RAS or CAS).

CP2K [8] is a package developed as a software for calculations that are relevant for solid state physics and quantum chemistry. Implementation of atomistic simulations for a list of systems, such as crystalline solids, liquid materials, and biological systems, is realized in the CR2K package. The framework of $\mathrm{CP} 2 \mathrm{~K}$ implies modeling methods such as Gaussian/plane wave (GPW) basis-set and density functional theory (DFT), along with methods that present support for the general structure of package. For CR2K, a list of such methods is accomplished; for instance, it includes semi-empirical methods, random phase approximation (RPA), the Møller-Plesset perturbation theory (MP2), the classical force fields, the density functional based tight binding (DFTB) method, the local-density approximations (LDAs), the generalized gradient approximation (GGA), and so on. Runs of simulations are also available in THE CR2K package, specifically, CR2K carries out simulations of Monte Carlo, molecular dynamics, metadynamics, and Ehrenfest dynamics; moreover, the package implies energy minimization, transition state optimization, core level spectroscopy, vibrational analysis, and so on.

CPMD [9] is a code that performs the implementation of density functional theory by using extremely well parallelized plane/pseudopotential wave for ab initio molecular dynamics. Using the Car-Parrinello molecular dynamics scheme, the CPMD code executes quantum molecular dynamics simulations limited to systems of a few hundred atoms. Moreover, the CPMD code is capable of a hybrid quantum mechanical and molecular mechanics interface, retaining practices from the GROMOS96 molecular dynamics code, in order to expand its domain of applicability to larger biologically relevant systems. It can also be used for the simulation of chemical reactions, particularly in the condensed phase. Moreover, with the restricted open-shell Kohn-Sham excited state gradient, it allows the molecular dynamics simulation of photoreactions. 
CRYSTAL [10] is a purpose program for the exploration of crystalline solids. The program carries out calculations on the electronic structure of Hartree-Fock periodic systems, various hybrid approximations, or density functional theory. In addition, the CRYSTAL program implements linear combinations of atom-centered Gaussian functions to expand the Bloch functions of the periodic systems. The calculations on restricted (closed shell) and unrestricted (spin-polarized) are feasible with all-electron and valence-only basis sets with effective core pseudo-potentials.

DACAPO [11] is a program designed for the implementation of calculations on total energy's system. The performance of calculations is realized by DACAPO through the usage of the camp atomic simulation environment (ASE). The base of the program is density functional theory (DFT). For the valence electronic states, DACAPO implies a plane wave basis; in addition, the description of the interactions of core-electron is realized by usage of Vanderbilt ultrasoft pseudo-potentials. The calculations on various generalized gradient approximation (GGA) exchange-correlation potentials and local density approximation (LDA) are carried out through program the accomplishment of state-of-the-art iterative algorithms. The solutions to the Schrodinger equations along with density functional theory allow implementing contemporaneously structural relaxation and molecular dynamics. The program implies compilation for parallel along with serial execution.

DALTON [12] is an ab initio chemistry program suitable for quantum calculations on distinct molecules properties. The realization of the program is carried out through the implementation of MCSCF, Hartree-Fock perturbation theory, MP2, and coupled cluster theories. The DALTON package is capable of automatic determination of a huge number of molecular properties based on a coupled cluster, density functional theory (DFT), MøllerPlesset perturbation theory (MP2), the Hartree-Fock (HF) method, or multi-configurational self-consistent field (MCSCF) reference wave function.

deMon2k [13] is a software package elaborated for calculations on DFT. At the core of the package, it is presented as an approach, based on the linear combination of Gaussiantype orbital (LCGTO), to the self-consistent solution of the Kohn-Sham (KS) DFT equations. The deMon2k program approaches the introduction of an auxiliary function basis for the variation fitting of the Coulomb potential. This allows avoiding the calculation of the four-center electron repulsion integrals.

DMol3 [14] is a software package that was developed based on density functional theory (DFT) in conjunction with a numerical radial function basis set. The prime focus of the package is presented as a conjunction of calculations on the surfaces, clusters, molecules, and solids properties of electronic structures. The package implies the availability of an application of lower-dimensional periodicity simulations or 3D periodic boundary conditions for crystalline solid materials or boundary conditions of gas phase; in addition, DMol3 applies, for quantum simulations of recently wetted surfaces and solvated molecules, the implementation of the COSMO solvation model as a conductor screening model. Geometry optimization and the search for saddle point along with realization of the calculation of electronic configuration properties, including or not the geometry constraints.

DP [15] is an open-source software package. It is specified mainly for physicists' implementation of $\mathrm{ab}$ initio time-dependent density functional theory (TD-DFT) with a linear-response on a plane wave basis set and frequency-reciprocal space. It allows calculating both dielectric spectra, such as inelastic electron energy-loss spectroscopy, X-ray scattering spectroscopy, and coherent inelastic X-ray scattering spectroscopy, and optical spectra, e.g., optical absorption, refraction index, and reflectivity. The range of systems lies in the gap from crystalline or periodic solids, to clusters, surfaces, atoms, and molecules. A nice feature of this software package is the implementation of adiabatic local-density approximation and non-local approximations (even neglecting or including local-field effects), as well as the random phase approximation.

ErgoSCF [16] is a quantum chemistry program. Large-scale self-consistent field calculations can be implemented by ErgoSCF. Linear scaling is achieved by modern techniques 
such as hierarchic sparse matrix algebra, efficient integral screening, fast multipole methods, and density matrix purification.

Exabyte.io [17] is a cloud-based digital platform for content research and development. The platform allows designing and building molecular models and running the stimulations.

FHI-aims package [18] (Fritz Haber Institute ab initio molecular simulations package) is a code package designed to perform computational molecular and materials science. FHI-aims maintains density functional theory and many-body perturbation theory for the realization of semi-local and hybrid exchange-correlation functionals. The FHI-aims is capable of calculations of an amount near to thousands of atoms and provides efficient usage for (ten) thousands of cores.

The FPLO [19] package is code that was developed for finite systems to solve the Kohn-Sham equations with free boundary or on a conditions regular lattice. Orbital polarization correction, the full-featured linearized augmented plane wave (LAPW) method implementations, generalized gradient approximation (GGA) functional, and the local spin density approximation (LSDA) are available and realized by the FPLO package, which is programmed for a local-orbital full-potential minimum-basis. Relativistic effects either are considered in scalar-relativistic or in full four-component formalism. A level comparable to the advanced level of numerical precision was achieved. Because of this high accuracy, implementation calculations on system's entire potential unit cells include up to 300 atoms; moreover, calculations can be carried out on uniprocessor machines.

GAMESS-UK [20] and GAMESS-US [21] (General Atomic and Molecular Electronic Structure System) are general-purpose computer software, designed for the computational chemistry program. GAMESS-UK and GAMESS (US) implement basic general calculations on computational chemistry, such as multiconfigurational self-consistent field, generalized valence bond, density functional theory, and the Hartree-Fock method. Estimation of correlation corrections after the self-consistent field calculations is realized by the approaching of second-order Møller-Plesset perturbation theory, coupled cluster theory, and configuration interaction. Calculations on relativistic corrections are estimated through the application of third-order Douglas-Kroll scalar terms. The solvent effect is treated by the usage of molecular mechanics and quantum mechanics through discrete effective fragment potentials or continuum models, such as the polarizable continuum model (PCM).

The Gaussian [22] package is a general-purpose software, developed for computational chemistry. The Gaussian package makes available electronic structure modeling with stateof-the-art capabilities. The implementation of Gaussian orbitals instead of Slater-type orbitals allows accelerating calculations on molecular electronic structure. The prediction of molecules' properties, such as Raman and IR spectra, molecular energies, vibrational frequencies, atomic charges, structures of transition states, NMR shielding, and magnetic susceptibilities, among others, is implied by Gaussian. Scientific and modeling feature are contained in the Gaussian package; moreover, calculations are not entangled with any artificial limitations, except for your computing resources and time.

GPAW [23] is a Python code for density-functional theory (DFT). GPAW is based on the atomic simulation environment (ASE) and the projector-augmented wave (PAW) method. Realization of the description of wave functions is done with real-space uniform grids, multigrid methods and the finite-difference approximation (FD), atom-centered basis-functions (LCAO), and plane-waves (PW). GPAW calculations are controlled through scripts written in the programming language Python. GPAW relies on the atomic simulation environment (ASE) that handles molecular dynamics, analysis, visualization, geometry optimization, and more.

HORTON [24] is a modular quantum chemistry program that allows to carry out various ab initio and DFT calculations following the post-processing options such as atomsin-molecule analysis including Hirshfeld, extended Hirshfeld, iterative Hirshfeld, Becke, and iterative stockholder; orbital entanglement analysis; and electrostatic potential fitting of atomic charges. 
HyperChem [25] is an environment that was developed for molecular modeling. The base features of HyperChem are presented in the program as a conjunction of 3D visualization and 3D animation with molecular dynamics, molecular mechanics, and quantum calculations on chemical systems. The program HyperChem is available on computers with Windows as well as Linux operative systems. The kinetics, spectra, and thermodynamics are implemented; moreover, geometry optimization, calculations in search of transition state, and application of modeling surfaces' potential energy are also realized by HyperChem. In addition, Langevin dynamics and metropolis Monte Carlo simulations are run in the environment. Calculations of free energies, heat capacities, and entropies are accomplished through treatment of vibrational partition, rotational, and translational functions.

JDFTx [26] is a code, developed for plane-wave density-functional theory (DFT), and specifically the code that supports of all the standard functionalities presented in electronic DFT software and several semi-local, meta-GGA, and EXX-hybrid exchangecorrelation functions, which are available with additional options by linking to the library of exchange-correlation functionals ( $\mathrm{LibXC}$ ). DFT $+\mathrm{U}$ is capable of approaching localized electrons. Including van der Waals interactions in calculations is possible in pair potential dispersion corrections. The usage of analytically continued energy functionals allows JDFTx to implement total energy minimization. Before performing the first electronic solution, following straight after the end of initialization, JDFTx carries prints out that present a list for optional features of current relevant citations of the code. JDFTx is based on C++ code, developed with an approach of highly templated and object-oriented functions. This was applied for the expression of all the physics that appear in the DFT++ algebraic framework; moreover, it allows to contemporaneously support a range of hardware architectures (such as graphics processing unit (GPU) using computed unified device architecture (CUDA)) and to maintain a small memory footprint, requiring any implementations for each architecture, especially hand-optimized.

The Maple Quantum Chemistry (MQC) package [27] is a toolbox, developed for the predictions, research, and design of novel molecules in an available and easy-to-build encirclement. The Maple Quantum Chemistry package presents a combination of up-todate quantum chemistry software, including advanced reduced density matrix (RDM) techniques, density functional theory (DFT), and wave function methods, with the mathematical calculations and approachability of the MQC package allowing to provide a complex, available for usage encirclement for the parallel computation of electronic energies and molecules' properties. Moreover, the MQC package uses a database that includes nearly 96 million molecules to define molecules; in addition, the package runs quantum computations with an approach of electronic structure methods, analyzes molecular energies, and carries out high-quality 2D and 3D plots and animations.

Molcas [28] is a quantum chemistry ab initio software, which presents itself as a package developed on a stable version of OpenMolcas. As the basic idea of code is an ability to consider general electronic structures, the focus of the package is primarily turned to multiconfigurational methods; therefore, the number of approaches is typically connected to the consideration of highly degenerate states.

MOLGW [29] is a Gaussian-type orbital (GTO) code designed to implement a selfconsistent mean-field calculation for finite systems (clusters, molecules, atoms) with a sequent application of a many-body perturbation theory on the system. The MOLGW code performs the approximation for the optical excitations applying the Bethe-Salpeter equation and, for the self-energy, the code accomplishes the density-functional theory (DFT) code for preparation of the subsequent many-body perturbation theory (MBPT) runs. MOLGW is capable of semi-local and standard local approximations of density-functional theory (DFT) along with several range-separated hybrid and hybrid functionals; moreover, the usage of a Gaussian-type orbitals basis set allows for the entire standard quantum chemistry tools to be reused. MOLGW is available for direct calculations on systems 
containing near to 100 atoms; larger calculations are feasible, but require computers that are more powerful.

Molpro [30] is a complex system composed of ab initio programs designed for calculations on molecular electronic structures. Molpro comprises programs that were well connected in parallel. Standard computational chemistry approaches include the multireference wave function methods, state-of-the-art high-level coupled-cluster, density functional theory (DFT) with a large choice of functionals, and so on. Molpro allows to implement electronically excited states by consideration of the complete active space selfconsistent field (CASSCF)/the multiconfigurational self-consistent field (MCSCF) methods, the complete active space second-order perturbation theory (CASPT2) method, the multireference configuration interaction (MRCI) method, or the full configuration interaction (FCI) methods, or by response methods such as the time-dependent density functional theory (TDDFT), coupled cluster methods (CC2), and equation-of-motion coupled cluster with single and double excitations (EOM-CCSD). Molpro accomplishes the modules that were developed for computing molecular properties such as further wave function analysis, calculations on harmonic as well as inharmonic vibrational frequencies, and geometry optimization. The analytical availability of energy gradients is carried out through the coupled cluster single-double (CCSD) method, the coupled cluster method for single and double excitations with exponential interelectronic distance explicit correlation correction (CCSD-F12), density functional theory (DFT), the Hartree-Fock (HF) method, the Møller-Plesset perturbation theory (MP2), the second-order Møller-Plesset perturbation theory with exponential interelectronic distance explicit correlation correction (MP2-F12), the quadratic configuration interaction with single and double excitations (QCISD), the quadratic configuration interaction with single and double excitations and triple excitations (QCISD(T)), the complete active space self-consistent field (CASSCF) method, and the complete active-space second-order perturbation theory (CASPT2). Approximations of density fitting (the density functional (DF) or the resolution-of-the-identity (RI) approximations) are able to accelerate the density functional theory (DFT) and Møller-Plesset perturbation theory (MP2) calculations that imply large basis sets and are clearly correlated with methods such as MP2-F12, CCSD(T)-F12, CASPT2-F12, and MRCI-F12. This allows to minimize errors that might appear owing to incompleteness of the basis set, thus achieving results near to CBS quality using the triple-zeta basis sets. An application of Molpro to large molecules with high accuracy is implemented by the combination of local approximations and efficient parallelization, high-level methods such as the accurate intermolecular interaction energies using explicitly correlated local coupled-cluster methods (PNO-LCCSD(T)-F12), and the pair natural orbital local second-order Møller-Plesset perturbation theory (PNO-LMP2-F12). Moreover, wave function in DFT (WF-in-DFT) calculation application or quantum mechanics/molecular mechanics (QM/MM) methods are available to be extended in the applicability as ab initio methods applied to systems of a large size that present biochemical and chemical interests.

MOPAC [31] (Molecular Orbital PACkage) is a quantum chemistry program based on Thiel's NDDO and Dewar approximation. The package allowed to use the improved handling of large biomolecules, including the ability to more easily manipulate macromolecules, e.g., to ionize and de-ionize individual atoms and residues, superimposition and calculation of RMSD for pairs of systems, and easier specification of individual atoms.

The Massively Parallel Quantum Chemistry (MPQC) [32] platform is a platform developed as a package for building simulation capable of run ab initio of the electronic structure of periodic solids and other molecules. The focus is directed on methods of manybody electronic structure, such as coupled-cluster (CC2) or the Møller-Plesset perturbation theory (MP2). The current (4th) version of the MPQC4 package presents as the original MPQC platform revision of the conceptual design. The package is able to use the Gaussian integrals library Libint embedding, distribution of task-based programming model, and runtime MADWorld, causing the massively parallel tensor framework TiledArray. 
MRCC [33] is a software that presents itself as a suite developed for quantum chemistry programs of $\mathrm{ab}$ initio and density functional with high-accuracy calculations on an electronic structure. MRCC's special feature is the performance of automated programming tools that are able to develop routines of tensor manipulation, which are not independent of the quantity of the corresponding tensors indices, thus the application of quantum chemical methods is generally simplified. The approach based on the automated tools of the program allows realizing techniques of high complexity for quantum chemistry models. The applied methods include and compose multi-reference CC approaches, arbitrary coupled-cluster (CC) taken as a reference and configuration interaction (CI) methods, arbitrary perturbative CC approaches, CC and CI energy derivatives, and response functions. In addition, the number of features that the package implies are capable of availability with relativistic Hamiltonians, which allows accurate calculations on heavy element systems. The techniques were developed specifically for cost-reduction on calculations; moreover, approaches with local correlation allow high-precision calculations on largeand medium-sized molecules.

NTChem [34] is a package that implies itself as a high-performance software developed in R-CCS from scratch. It is specified explicitly for the computation for general purpose of the molecular electronic structure calculation. NTChem presents a complex new software of ab initio quantum chemistry; moreover, it contains standard quantum chemistry and original applications.

NWChem [35] is a software package for ab initio computational chemistry, and was designed in a way to include both molecular dynamics and quantum chemical functionality at the same time. The prime focus of NWChem remains to run on parallel supercomputers with high-performance along with conventional workstation clusters. The aims of the package are to be scale both in its usage of available parallel computing resources, and in its ability to consider efficiently problems.

ONETEP [36] (Order-N Electronic Total Energy Package) is a linear-scaling code based on density functional theory (DFT). It was developed explicitly for calculations on quantum-mechanical systems. The prime focus of ONETEP is to improve and apply the overall linear scaling and to control accuracy, keeping it high. The optimization procedures and the density-matrix formulation of density functional theory (DFT) were both included in the method of code; therefore, the description of the local orbitals or non-orthogonal generalized Wannier functions and for the density-kernel is implemented. The approaches of these methods to a great number of systems are able to demonstrate the performance of the realization prime focus of ONETEP.

OpenAtom [37] is an ab initio software for molecular dynamics; being a parallel simulation, it allows to study atomic along with molecular systems that are based on principles of quantum chemical. The main approach of the software is the CPAIMD algorithm that runs calculations on the forces acting independently on each atom as the result of the summation of multiple terms determined by plane-wave density functional solutions. OpenAtom implies research on complex systems, such as atomic systems and electronic physics in semiconductor, metallic, biological, and other molecular systems' implementation; in addition, OpenAtom is programmed on top of Charm++ that is a parallel framework for programming based on an over-decomposition.

OpenMx [38] is an open source software that allows estimation of a wide variety of advanced multivariate statistical models. OpenMx consists of a library of functions and optimizers that allow a quick and flexible definition of an SEM model and estimation of parameters given the observed data.

ORCA [39] is a program package that implies a general purpose tool for quantum chemistry. ORCA contains a number of modern methods for calculations on electronic structure, such methods as the semi-empirical quantum chemistry methods, the multireference methods, the coupled cluster (CC2), the many-body perturbation theory (MBPT), the density functional theory (DFT), and so on. The prime focus of ORCA is directed to the availability of applying the calculations to transition metal complexes and their 
optical properties, large molecules, and others. As a great amount of attention in the ORCA package is paid to spectroscopic open-shell molecules' properties, the package implies an extensive list of standard quantum chemical methods for this issue. The methods range from single- and multireference correlated ab initio methods and the consideration of ambient and relative effects to semi-empirical methods and density functional theory (DFT).

PARSEC [40] is a package that implements the realization of dentinal functional theory (DFT) calculations on solids and molecules. The code implies non-periodic boundary conditions without the performance of super-cells; moreover, the code accomplishes an equal well handling with periodic boundary and periodic conditions. PARSEC is programmed capable of an easy amenable process for efficient massive parallelization that performs highly effective calculations on far more than large systems.

PSI4 [41] is an ab initio quantum chemistry program that was programmed as a hybrid of $\mathrm{C}++$ and Python. The main focus of the PSI4 program is the performance of calculations on an electronic structure. PSI4 implies realization of the density functional theory (DFT), the coupled cluster (CC) theory, the Hartree-Fock (HF) method, the configuration interaction (CI), the many-body perturbation theory (MBPT), the density cumulant theory (DCT), the symmetry-adapted perturbation theory (SAPT), and so on.

PyQuante [42] is a software that was developed as a suite for programs that imply Gaussian-type orbital (GTO) basis sets for implementation of quantum chemistry methods. The basic code, which is presented in PyQuante, is programmed in a way such that it is easily comprehended and easily modified, despite that it is time-consuming. The software provides a set of tools that can be used by scientists to construct self-developed quantum chemistry programs without the necessity of writing such things as every low-level routine. PyQuante might be suitable for educational purposes for students who are involved in a quantum and computational chemistry development.

PySCF [43] (Python-based Simulations of Chemistry Framework) is a Python/Cbased package of a number of open-source compositions of electronic structure module development. PySCF implies a platform that is specified on the calculations on quantum chemistry systems and on the development of methodology. The package is capable of simulation of molecules' and crystals' properties. The PySCF provides Hamiltonians with post-mean-field and mean-field methods. Extensibility is implied and implemented by the fact that almost all of the PySCF features are realized in Python, whereas parts critical to the calculation are programmed in C; therefore, the combination of Python/C allows the package to be complete efficiently and to accomplish C- or FORTRAN-based quantum chemistry programs.

QMCPACK [44] (Quantum Monte Carlo PACKage) is a package designed for ab initio calculations on an electronic structure. QMCPACK implies computations of such systems as model Hamiltonians, atoms, molecules, and insulating and metallic solids. The package contains real space quantum Monte Carlo algorithms with embedded diffusion and reputation. Slater-Jastrow type trial wave functions are implemented as one with a complicated optimizer that is programmed in a way of an opportunity to improve thousands and tens thousands of parameters. QMCPACK carries out the quantum Monte Carlo method for the orbital space auxiliary-field; moreover, approaching cross validation between different highly accurate methods allows for optimization of the realization of calculations. A large number systems of electrons is currently available on high-performance computers; in particular, such calculations are relevant for graphical processing unit systems and multicore central processing units.

Quantum ESPRESSO [45] (opEn-Source Package for Research in Electronic Structure, Simulation, and Optimization) is a suite developed for computer codes to accomplish calculations on an electronic structure and modulations of nanoscale materials. The general framework of Quantum ESPRESSO is based on the density functional theory (DFT), pseudopotentials (ultrasoft and norm conserving), the density functional perturbation theory, and plane waves basic sets. 
RMG [46] is an extraordinary example of highly scalable DFT electronic structure code, which is developed for solutions of Kohn-Sham equations right on a 3D real space grid without including the usage of basis set functions.

SAMSON [47] is the platform specified on integrated molecular design. It allows to model nanosystems through their structures, dynamics, interactions, visuals, and properties.

SIESTA [48] is a computer program implementation that allows performing calculations on electronic structures and on molecular dynamics simulations for solids and molecules. The program implies the usage of strictly localized basis sets to improve efficiency; in addition, the realization of algorithms based on linear scaling allows to apply SIESTA to huge systems. The feature observed in the code presents itself with accuracy. The cost of application is placed in a wide tern ranging from simulations with high accuracy, matching the quality of a number of other approaches, to quick exploratory calculations, for instance, all-electron and plane-wave methods.

VOTCA-XTP [49] is an open-source library that was programmed for calculations on the organic material properties of electronic structures, such as excited state properties through the GW plus Bethe-Salpeter equation (GW-BSE) method, spectra usage of the quantum mechanics/molecular mechanics (QM/MM) approach, along with excited state consideration with GW-BSE. Calculations on hole and electron mobilities were realized using electronic couplings and kinetic Monte Carlo. Usage of the Monte Carlo kinetic and electronic couplings allows to perform singlet, electron, triplet, and hole diffusion constants. Energetic disorder for electrons or holes or excitons was implemented along with the quantum mechanics/molecular mechanics (QM/MM) or molecular mechanics multipole methods.

Yambo [50] is a software package designed for the implementation of calculations, based on many-body perturbation theory (MBPT) methods (such as GW and BSE) and time-dependent density functional theory (TDDFT), for molecule systems and crystalline solid materials. Yambo implies calculations on the excited state properties of physical systems; moreover, the prediction of fundamental properties, such as defect quasi-particle energies, band gaps of semiconductors, optics, and so on, is accurate.

Thus, we call on the scientific community to pay attention to this software for computer modeling in chemistry and related areas of natural sciences and to widely use these program packages in daily research work.

Author Contributions: Conceptualization, A.S.N.; methodology, A.S.N.; software, M.V.M. and A.S.N.; validation, M.V.M. and A.S.N.; formal analysis, M.V.M. and A.S.N.; investigation, M.V.M. and A.S.N.; resources, M.V.M. and A.S.N.; data curation, M.V.M. and A.S.N.; writing-original draft preparation, M.V.M. and A.S.N.; writing-review and editing, A.S.N.; visualization, M.V.M. and A.S.N.; supervision, A.S.N.; project administration, A.S.N.; funding acquisition, this review was written without attracting additional external funding from any scientific foundations. All authors have read and agreed to the published version of the manuscript.

Funding: This review was written without attracting additional external funding from any scientific foundations.

Data Availability Statement: Not applicable.

Conflicts of Interest: The authors declare no conflict of interest.

\section{References}

1. ABINIT. Available online: https://www.abinit.org/ (accessed on 24 September 2021).

2. ACES. Available online: http:/ / www.qtp.ufl.edu/ACES/ (accessed on 24 September 2021).

3. BerkeleyGW. Available online: https:// berkeleygw.org/ (accessed on 24 September 2021).

4. BigDFT. Available online: https:/ / bigdft.org/ (accessed on 24 September 2021).

5. BrianQC and Q-Chem. Available online: https://www.brianqc.com/ and https://www.q-chem.com (accessed on 24 September 2021).

6. CASTEP. Available online: http:/ / www.castep.org/CASTEP/CASTEP (accessed on 24 September 2021).

7. COLUMBUS. Available online: https://www.univie.ac.at/columbus/ (accessed on 24 September 2021).

8. CP2K. Available online: https://www.cp2k.org/ (accessed on 24 September 2021). 
9. CPMD. Available online: https://bioexcel.eu/software/cpmd/ (accessed on 24 September 2021).

10. CRYSTAL. Available online: https://www.crystal.unito.it/index.php (accessed on 24 September 2021).

11. Dacapo. Available online: https://wiki.fysik.dtu.dk/dacapo/dacapo (accessed on 24 September 2021).

12. Dalton and LSDalton. Available online: https:/ / daltonprogram.org/ (accessed on 24 September 2021).

13. deMon2k. Available online: http://www.demon-software.com/public_html/index.html (accessed on 24 September 2021).

14. $\mathrm{DMol}^{3}$. Available online: http://dmol3.web.psi.ch/dmol3.html (accessed on 24 September 2021).

15. The DP code. Available online: http://www.dp-code.org/ (accessed on 24 September 2021).

16. ErgoSCF. Available online: http://www.ergoscf.org/ (accessed on 24 September 2021).

17. EXABYTE.IO. Available online: https:/ / exabyte.io/ (accessed on 24 September 2021).

18. FHI-aims. Available online: https://aimsclub.fhi-berlin.mpg.de/ (accessed on 24 September 2021).

19. FPLO. Available online: https:/ / www.fplo.de/ (accessed on 24 September 2021).

20. GAMESS-UK. Available online: https:/ / computingforscience.com/gamess-uk/index.shtml (accessed on 24 September 2021).

21. GAMESS. Available online: https://www.msg.chem.iastate.edu/ (accessed on 24 September 2021).

22. Gaussian. Available online: https://gaussian.com/ (accessed on 24 September 2021).

23. GPAW. Available online: https://wiki.fysik.dtu.dk/gpaw/ (accessed on 24 September 2021).

24. HORTON. Available online: https:/ theochem.github.io/horton/ (accessed on 24 September 2021).

25. HyperChem. Available online: https://www.chemits.com/en/software/molecular-modeling/hyperchem/ (accessed on 24 September 2021).

26. JDFTx. Available online: https://jdftx.org/ (accessed on 24 September 2021).

27. Maple Quantum Chemistry Toolbox. Available online: https://www.maplesoft.com/products/toolboxes/quantumchemistry/ (accessed on 24 September 2021).

28. Molcas. Available online: https://www.molcas.org/ (accessed on 24 September 2021).

29. MOLGW. Available online: http:/ /www.molgw.org/ (accessed on 24 September 2021).

30. Molpro. Available online: https://www.molpro.net/ (accessed on 24 September 2021).

31. MOPAC. Available online: http:/ / openmopac.net/ (accessed on 24 September 2021).

32. MPQC. Available online: https://mpqc.org/ (accessed on 24 September 2021).

33. MRCC. Available online: https://www.mrcc.hu/ (accessed on 24 September 2021).

34. NTChem. Available online: https://www.r-ccs.riken.jp/software_center/software/ntchem/overview/ (accessed on 24 September 2021).

35. NWChem. Available online: https://nwchemgit.github.io/ (accessed on 24 September 2021).

36. ONETEP. Available online: https://www.onetep.org/ (accessed on 24 September 2021).

37. OpenAtom. Available online: https://charm.cs.illinois.edu/OpenAtom/doxy/html/index.html (accessed on 24 September 2021).

38. OpenMx. Available online: https:// openmx.ssri.psu.edu/ (accessed on 24 September 2021).

39. ORCA. Available online: https:// orcaforum.kofo.mpg.de/app.php/portal (accessed on 24 September 2021).

40. PARSEC. Available online: https://parsec.oden.utexas.edu/ (accessed on 24 September 2021).

41. PSI4. Available online: https://psicode.org/ (accessed on 24 September 2021).

42. PyQuante. Available online: http:// pyquante.sourceforge.net/ (accessed on 24 September 2021).

43. PySCF. Available online: https://pyscf.org/ (accessed on 24 September 2021).

44. QMCPACK. Available online: https:/ / qmcpack.org/ (accessed on 24 September 2021).

45. Quantum ESPRESSO. Available online: https://www.quantum-espresso.org/ (accessed on 24 September 2021).

46. RMG. Available online: http:/ / www.rmgdft.org/ (accessed on 24 September 2021).

47. SAMSON. Available online: https://www.samson-connect.net/ (accessed on 24 September 2021).

48. SIESTA. Available online: https:/ / siesta-project.org/siesta/ (accessed on 24 September 2021).

49. VOTCA-XTP. Available online: https://github.com/votca/xtp (accessed on 24 September 2021).

50. YAMBO. Available online: http:/ / www.yambo-code.org/ (accessed on 24 September 2021). 